\title{
Gengivite Ulcerativa Necrosante: um relato de caso
}

\author{
Necrotizing Ulcerative Gingivitis: A Case Report
}

\author{
Letícia Regina Morello Sartori ${ }^{1}$ \\ Kauana Ferreira ${ }^{2}$ \\ Thiago Marchi Martins ${ }^{3}$ \\ Marcos Britto Correa ${ }^{4}$ \\ Luiz Alexandre Chisini ${ }^{5}$
}

\section{RESUMO}

Introdução: Diversos fatores predisponentes são reportados na Gengivite Ulcerativa Necrosante (GUN): estresse, deficiências nutricionais e disfunções do sistema imunológico.

Objetivo: Descrever e discutir a abordagem diagnóstica e o manejo terapêutico conservador de um caso clínico de GUN em um paciente masculino de 21 anos, sem doença sistêmica e com o estresse como provável mecanismo de patogênese predisponente envolvido.

Caso Clínico: O paciente procurou atendimento devido a dor na gengiva e halitose. Ao exame clínico observou-se ausência de papila interdental com ulcerações, secreção supurativa e sangramento ao toque. Ao questionado reportou estar mais estressado que o habitual, dormindo pouco e alimentando-se de forma menos saudável que de costume. Assim, foi realizado o debridamento local. $O$ usuário foi orientado a realizar bochechos com água oxigenada (dez volumes). Aconselhamento dietético e a indicação de realização de atividades físicas e melhora da qualidade e quantidade de sono também foram feitas. Após um mês, foi obtida a resolução dos sinais e sintomas e nenhuma recidiva foi observada em 1 ano de acompanhamento. Conclusão: Um correto diagnóstico dos fatores causais é fundamental para a formulação de um plano de tratamento preciso. A terapia inicial é realizada com o objetivo de interromper a progressão da doença e diminuir os sintomas clínicos da doença. Além disso, o projeto terapêutico deve ser direcionado para uma abordagem ampliada do sujeito, a qual deve visar o controle dos fatores predisponentes.

Palavras Chaves: Periodontia, Gengivite Ulcerativa Necrosante, Caso Clínico, Gengivite, Periodontite

\begin{abstract}
Introduction: Several predisposing factors are reported in Necrotizing Ulcerative Gingivitis (GUN): stress, nutritional deficiencies, and immune system dysfunction.

Objective: To describe and discuss the diagnostic approach and conservative therapeutic management of a clinical case of GUN in a 21-year-old male patient with no systemic disease and with stress as a probable mechanism of predisposing pathogenesis involved.

Clinical Case: The patient sought care due to gum pain and halitosis. Clinical examination showed absence of interdental papilla with ulceration, suppurative secretion and bleeding to the touch. The respondent reported being more stressed than usual, sleeping little and eating more unhealthy aliments than usual. Thus, the local debridement was performed. The patient was instructed to perform mouthwash with hydrogen peroxide (ten volumes). Dietary counseling and indication of physical activity and improvement of sleep quality and quantity were also made. After a month, the resolution of the signs and symptoms was obtained, and no relapse was observed at 1 year of follow-up.

Conclusion: A correct diagnosis of causal factors is essential for the formulation of an accurate treatment plan. Initial therapy is aimed at stopping the progression of the disease and reducing the clinical symptoms of the disease. In addition, the therapeutic design should be directed towards an extended approach of the subject, which should aim at the control of the predisposing factors.

Key-words: Periodontitis, Necrotizing Ulcerative Gingivitis, Clinical Case, Gingivitis, Periodontitis

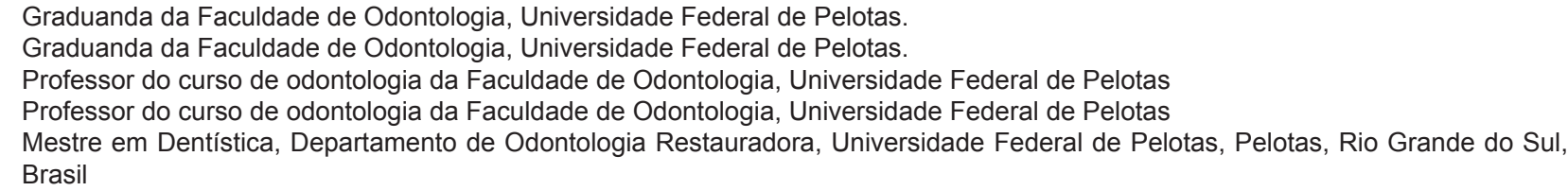




\section{INTRODUÇÃO}

A Gengivite Ulcerativa Necrosante (GUN) é uma forma pouco prevalente doença periodontal. ${ }^{1}$ Ela é conhecida há séculos e tem sido identificada por diversos nomes, dentre eles, doença de Vincent, gengivite ulcerativa aguda e boca de trincheira (devido à elevada prevalência entre soldados da primeira guerra mundial que estavam expostos à grande estresse psicológico, pobre higiene bucal e má nutrição). A GUN apresenta uma prevalência baixa na população mundial (menor que $1 \%$ ), 1 embora alguns estudos em populações específicas (Síndrome da Imunodeficiência Humana (HIV) infectados e soldados) têm apontado que ela pode acometer até $11 \%$ destes indivíduos. ${ }^{2-5}$ Além disso, adultos jovens parecem ser os indivíduos mais afetados por esta patologia. ${ }^{2-5}$

A GUN manifesta-se clinicamente com quadros agudos de rápida necrose e destruição do tecido gengival interdental. A etiologia da GUN é complexa, sendo relacionada basicamente a uma agressão inicial de origem bacteriana e uma resposta imune alterada do hospedeiro. ${ }^{1,5-7}$ Bactérias Gram negativas são apontadas como as principais agressoras inicias. ${ }^{41,6,8-13}$ Assim, algumas características clínicas frequentemente são observadas durante o exame clínico: necrose das papilas interdentais, hemorragia, supuração, formação de membranas acinzentadas ou fibrinoporulentas sobre as lesões, além de odor fétido característico. Em alguns casos, sintomas de ordem geral como linfadenopatia, mal-estar e febre podem estar presentes, apresentando rápida progressão. ${ }^{4,5,8,9} \mathrm{Em}$ geral, o acúmulo de placa bacteriana e de cálculo dental não justifica tamanha inflamação dos tecidos gengivais, fator esse que pode ajudar no diagnóstico diferencial da doença com as demais formas de genvivites. ${ }^{5}$

Além disso, diversos fatores predisponentes - os quais alteram a capacidade imunológica
- são reportados: estresse, deficiências nutricionais (faltas vitamínicas como $\mathrm{A}$, B2 e, principalmente, a vitamina C estão relacionadas à doença $1,2,10$ ), disfunções do sistema imunológico (especialmente a infecção pelo HIV) e pobre higiene bucal. ${ }^{41,6,8-16}$ Desta forma, um correto diagnóstico dos fatores causais e predisponentes é fundamental para a formulação de um plano de tratamento preciso.

Recentemente, o estresse tem sido diretamente implicado no comprometimento do sistema imunológico do corpo sendo um importante fator predisponente à GUN, explicado por dois mecanismos. ${ }^{17-20} \mathrm{O}$ primeiro relacionado à uma depressão em componentes celulares, principalmente de leucócitos polimorfonucleados e na capacidade de quimiotaxia e fagocitose. Assim, o estresse parece alterar o processo inflamatório dificultando a cicatrização devido ao fato de deprimir as funções dos neutrófilos e linfócitos, o que facilita a invasão e bacteriana. ${ }^{21-24}$ Além disso, o segundo fator está relacionado à diminuição da microcirculação gengival e do fluxo salivar. ${ }^{17}$, ${ }^{20} \mathrm{~A}$ resistência do tecido hospedeiro pode ser alterada por mecanismos que atuam por meio do sistema nervoso autônomo e de glândulas endócrinas, resultando em elevação dos níveis de cortisol e catecolaminas. Isso pode reduzir a microcirculação gengival e o fluxo salivar, aumentando a colonização bacteriana e favorecendo o estabelecimento ou progressão da GUN. ${ }^{25,26}$

Como tratamento, deve-se formular um plano terapêutico que vise a eliminação ou diminuição dos fatores predisponentes assim como a realização de raspagem e alisamento coronário e radicular e debridamento das lesões, quando estas estiverem na fase aguda da doença. Além disso, a prescrição de analgésicos, bochechos com água oxigenada a 10 volumes ou clorexidina a $0.12 \%$ e antibioticoterapia podem ser prescritas em 
casos com sintomatologia sistêmica. 2, 60 correto tratamento leva à uma recuperação do periodonto de proteção em poucos dias e o controle correto proporciona ao paciente menor número de possíveis recidivas e um menor número de sequelas, como a perda da papila dental e retração gengival. ${ }^{4,9}$

Neste contexto, o manejo ampliado e holístico dos indivíduos que apresentam GUN deve ser realizado considerando todos fatores que podem ser coadjuvantes às manifestações orais da doença, como por exemplo o estresse. No entanto, a maioria dos relatos de casos não apresentam esta abordagem ampliada, detendo-se basicamente às manifestações clínicas e ao manejo da doença ${ }^{27,28}$ ou relacionando a GUN com infecção por HIV. ${ }^{29}$, 30 Assim, este relato de caso descreve a abordagem diagnóstica e o manejo terapêutico conservador e ampliado de um caso clínico de GUN em um paciente masculino, de 21 anos, melanoderma, sem doença sistêmica e com o estresse como provável mecanismo de patogênese predisponente envolvido.

\section{Relato do CASO}

Paciente de 21 anos, sexo masculino, melanoderma, estudante universitário, chegou ao à Faculdade de Odontologia da Universidade Federal de Pelotas com queixa de dor e desconforto muito grande na gengiva. Reportou que isso ocorria há pelo menos uma semana. Além disso, relatou dificuldade para higienização e alimentação. Durante a entrevista dialogada, reportou não ter doenças sistêmicas e nem estar em tratamento médico ou fazendo uso de algum tipo de medicação. Não referiu ter alergias, problemas de cicatrização ou ser tabagista.

No exame clínico extrabucal nenhuma alteração (tal como linfadenopatia) foi encontrada. No entanto, durante a realização do exame intrabucal, foi observado alterações de tecidos gengivais. Nas regiões de gengiva interdental dos elementos anteroinferiores (principalmente entre os elementos $42 \mathrm{e}$ 43) e na região dos dentes 35 e 36 e distais de 47 e 37 foram observadas regiões de necrose (Figura 1), inversão da papila Inter dentária com ulcerações, exsudato purulento e sangramento ao toque, de forma semelhante à casos observados na literatura relacionados à GUN. ${ }^{27}, 29,30$ Além disso, ocorreu intensa sintomatologia dolorosa ao toque e odor fétido característico. Pequena quantidade de placa visível foi observada em comparação com a elevada destruição tecidual. Neste contexto, a suspeita diagnóstica foi de GUN. 1, 17, 27, 29, 30

Desta forma, uma profunda investigação dos fatores predisponentes foi realizada. Direcionando o diálogo, o paciente reportou estar em semana de provas na universidade, estando mais estressado que o habitual. Além disso, quando questionado sobre a sua alimentação, relatou estar se alimentando de forma menos saudável que de costume, além de ter diminuído às horas de sono nas últimas semanas.

Após ser realizado o diagnóstico de Gengivite Ulcerativa Necrosante com base nas características clínicas e na anamnese ${ }^{17}$ foi realizado, na mesma consulta, o debridamento local. Além disso, o usuário foi orientado a realizar bochechos com água oxigenada a dez volumes diluída em três partes de água, 3 vezes ao dia por 3 dias. Para investigação de possíveis alterações sistêmicas como deficiência vitamínica e HIV, foi solicitado ao usuário, nesta mesma sessão, a realização de exames de sangue para investigação sobre possíveis doenças de base como, por exemplo HIV, assim como para vitaminas do complexo $B, C$ e A. Além disso, o estudante foi instruído a retomar às horas habituais de sono (8 horas de sono por noite), ${ }^{31}$ assim como foi realizado um aconselhamento dietético. Para diminuição 

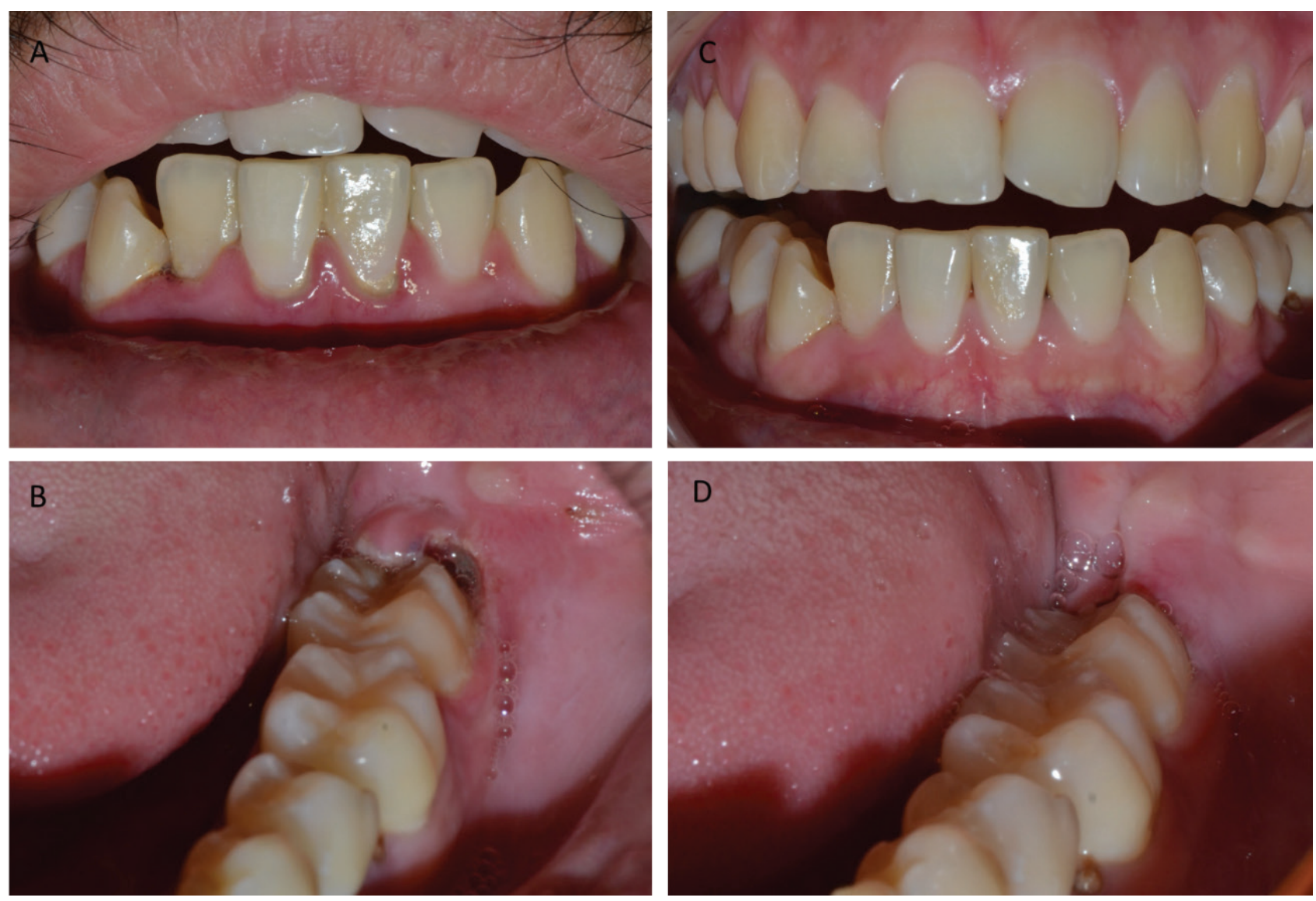

Figura 1 - Aspecto inicial e após 7 dias de tratamento da Gengivite Ulcerativa Necrosante. Na imagem (A) observamos alterações nas papilas interdentais com formação de membrana fibrinoporulenta na gengiva interdental entre os elementos 42 e 43. Em (B) observamos na região do dente 35 e distal do 37, locais de necrose tecidual. Em (C) e em (D) observamos a remissão da GUN após 7 dias de tratamento com água oxigenada 10 volumes.

do estresse, o usuário foi aconselhado a realizar atividades físicas regulares ${ }^{32}$ assim como uma possível busca por aconselhamento psicológico. ${ }^{33}$

Uma semana depois retornou para acompanhamento. Ele relatou ter realizado o uso dos bochechos, ter aumentado o número de horas de sono, assim como, ter melhorado a qualidade da dieta (alimentando-se com mais frutas e verduras e diminuindo a ingestão de açúcares refinados), embora não tenha aderida ao aconselhamento sobre iniciar atividades físicas. Ao exame clínico, as lesões já se encontravam menores e com menor sintomatologia dolorosa. Após duas semanas do diagnóstico, a região interdental entre os elementos 42 e 43 já se encontrava saudável novamente. $\mathrm{Na}$ região interdentária dos dentes 35 e 36 persistiu por mais uma semana. Após um mês, o usuário retornou com os exames solicitados, sendo negativo o de HIV e dentro da normalidade para os vitamínicos, reforçando a suspeita inicial de que o estresse possa ter sido o principal agente predisponente envolvido na patogênese da doença. Após um ano de acompanhamento, nenhuma recidiva da GUN foi observada.

\section{Dıscussão}

A Gengivite Ulcerativa Necrosante é uma doença inflamatória de carácter agudo que, na 
grande maioria dos casos, afeta a qualidade de vida do paciente..$^{1,5}$ Muitas vezes, essa doença afeta também a higienização bucal e a ingesta dealimentos. Destaforma, os indivíduos tendem a alimentar-se com alimentos menos nutritivos e com maior quantidade de açúcar, devido à sensação dolorosa de ingerir alimentos mais fibrosos. Além disso, a higiene também pode ser dificultada pela sintomatologia dolorosa, sangramento e/ou supuração. Desta forma, desencadeando um ciclo que pode culminar no agravamento do quadro clínico inicial, caso não seja tratado. ${ }^{5,16}$

Entre as espécies bacterianas que exercem estímulo sobre as estruturas periodontais, a literatura aponta para diferentes espécies, entre elas a Bacterioides, espiroquetas e $P$. Intermedia. ${ }^{13,34}$ Por muito tempo, o único fator a ser considerado na patogenia era a espécie bacteriana. ${ }^{11}$ Porém, hoje, se reconhece que a evolução da doença tem como fator determinante a resposta imune do indivíduo. ${ }^{5}$, 12 Quando estimulados, os leucócitos polimorfonucleares exercem efeito sobre o tecido por meio da produção de citocinas e imunomediadores, sendo esta relação a principal determinante do dano tecidual. ${ }^{12}$

Dentre os fatores apontados como influenciadores da patogênese da GUN, o estresse (junto com o HIV) são os fatores mais comuns. ${ }^{1}, 35,36 \mathrm{O}$ estresse afeta a resposta imune do indivíduo ${ }^{20}$ através do aumento do nível sanguíneo de cortisol, alterando assim, a capacidade de resposta e comportamento metabólito dos tecidos. Esse fato explica a alta prevalência e incidência de casos em pessoas que estão sujeitas a grande estresse psicológico, ${ }^{20}$ sejam estudantes em véspera de provas ou soldados em forças armadas. ${ }^{30}$, 37, 38 Tendo em vista que estudos têm apontado que a realização de atividade física poderia diminuir os níveis de estresse dos indivíduos, 32 assim como, melhorar a qualidade do sono, ${ }^{39}$ a indicação desta possibilidade para o usuário foi realizada. No entanto, o usuário não aderiu à esta estratégia terapêutica. Por outro lado, ele realizou a mudança sugerida na dieta e elevou a quantidade de horas de sono, uma vez que estas mudanças podem diminuir os níveis de cortisol e, consequentemente, o estresse. ${ }^{31}$

Alguns mecanismos pelos quais alimentos com grande quantidade de açúcares podem exercer efeitos adversos são devidos ao aumento no nível de glutationa-6desidrogenase, de ácido gama-aminobutírico (GABA), glutamato e alteração da dopamina nas ondas cerebrais. Assim, eles podem aumentar o estresse oxidativo nas células cerebrais por uma diminuição da monoamina oxidase e acetilcolina, a qual influência também a qualidade e a duração do sono. 31 De forma semelhante, deficiências de vitaminas do complexo $\mathrm{B}, \mathrm{Ae}$ - principalmente - C são apontadas como importantes para a patogênese da GUN por afetar o ciclo celular de células do epitélio, síntese de colágeno e a atividade fagocítica das células de defesa. ${ }^{1}$ Desta forma, o conhecimento e controle destes fatores deve ser levado em consideração durante a formulação do plano de tratamento.

Em relação ao tratamento da GUN, não existe um consenso. De forma geral, os tratamento se baseiam na terapêutica dos fatores predisponentes e na remoção do estímulo local e tratamento das lesões. ${ }^{4} \mathrm{~A}$ presença de placa e cálculo no local pode agravar o quadro clínico, devendo ser realizado o debridamento mecânico para esta finalidade. É importante, neste momento, levar em consideração a tolerância do paciente ao procedimento e a possibilidade de anestesia local. ${ }^{1,2}$ Além disso, a instrução de higiene bucal, prescrição de antibióticos, analgésicos e enxaguatórios bucais, assim como a orientação para cessar o uso de tabaco, são reportados na literatura. ${ }^{2} \mathrm{O}$ antibiótico de escolha para o tratamento da GUN é o Metronidazol 200mg, 
embora o tratamento da GUN com o uso de água oxigenada ou clorexidina em detrimento ao uso de antibióticos também parece ser eficaz. 1, 2,6 No presente caso, a utilização de água oxigenada apresentou um bom resultado já na primeira semana. Tal tratamento é de fácil realização e tem apresentado bons resultados, podendo ser a opção terapêutica de casos de pequena e média destruição tecidual. 1, 2,6

\section{Considerações finaIS}

Apesar do grande desconforto causados ao paciente, a Gengivite Ulcerativa Necrosante pode ser tratada de forma simples e eficiente restabelecendo a saúde periodontal. O correto diagnóstico dos fatores predisponentes é um dos pontos chaves para o sucesso da terapia.

\section{REFERÊNCIAS}

1. Dufty, J., Gkranias, N., Donos, N. Necrotising Ulcerative Gingivitis: A Literature Review. Oral Health Prev Dent. 2017;15(4):321-7. https:// www.ncbi.nlm.nih.gov/pubmed/28761942

2. Dufty, J., Gkranias, N., Petrie, A., McCormick, R., Elmer, T., Donos, N. Prevalence and treatment of necrotizing ulcerative gingivitis (NUG) in the British Armed Forces: a case-control study. Clin Oral Investig. 2017;21(6):1935-44. https:// www.ncbi.nlm.nih.gov/pubmed/27830369

3. Lopez, R., Fernandez, O., Jara, G., Baelum, $\mathrm{V}$. Epidemiology of necrotizing ulcerative gingival lesions in adolescents. J Periodontal Res. 2002;37(6):439-44. https://www.ncbi.nlm.nih. gov/pubmed/12472838

4. Mizrahi, Y. [NUG--necrotizing ulcerative gingivitis: a review]. Refuat Hapeh Vehashinayim (1993). 2014;31(3):41-7, 62. https://www.ncbi. nlm.nih.gov/pubmed/25219100

5. Carranza, F. Carranza's clinical periodontology. 12th edition. 2015

6. Herrera, D., Alonso, B., de Arriba, L., Santa Cruz, I., Serrano, C., Sanz, M. Acute periodontal lesions. Periodontol 2000. 2014;65(1):149-77. https://www.ncbi.nlm.nih. gov/pubmed/24738591
7. Schmidt, J., Weigert, M., Leuschner, C., Hartmann, H., Raddatz, D., Haak, R., et al. Active matrix metalloproteinase-8 and periodontal bacteria-interlink between periodontitis and inflammatory bowel disease? J Periodontol. 2018;89(6):699-707. https://www.ncbi.nlm.nih. gov/pubmed/29574823

8. Rowland, R. W. Necrotizing ulcerative gingivitis. Ann Periodontol. 1999;4(1):65-73. https:// www.ncbi.nlm.nih.gov/pubmed/10863376

9. Bascones-Martinez, A., Escribano-Bermejo, M. [Necrotizing periodontal disease: a manifestation of systemic disorders]. Med Clin (Barc). 2005;125(18):706-13. https://www.ncbi.nlm.nih. gov/pubmed/16324485

10. Todescan, S., Nizar, R. Managing patients with necrotizing ulcerative periodontitis. Managing patients with necrotizing ulcerative periodontitis. J Can Dent Assoc. 2013;79:d44 https:// www.ncbi.nlm.nih.gov/pubmed/23763731 11. Feller, L., Khammissa, R.A., Wood, N.H., Meyerov, R., Pantanowitz, L., Lemmer, J. Oral ulcers and necrotizing gingivitis in relation to HIV-associated neutropenia: a review and an illustrative case. AIDS Res Hum Retroviruses. 2012;28(4):346-51 https://www.ncbi.nlm.nih. gov/pubmed/?term=Oral+ulcers+and+necrotizi $n g+$ gingivitis+in+relation+to+HIV-associated $+n$ eutropenia\%3A+a+review+and+an+illustrative +case

12. Goncalves, L.S., Goncalves, B.M., Fontes, T.V. Periodontal disease in HIV-infected adults in the HAART era: Clinical, immunological, and microbiological aspects. Arch Oral Biol. 2013;58(10):1385-96. https://www.ncbi.nlm.nih. gov/pubmed/23755999

13. Sangani, I., Watt, E., Cross, D. Necrotizing ulcerative gingivitis and the orthodontic patient: a case series. J Orthod. 2013;40(1):77-80. https:// www.ncbi.nlm.nih.gov/pubmed/23524550

14. Chisini, L.A., Cademartori, M.G., Francia, A., Mederos, M., Grazioli, G., Conde, M.C.M., et al. Is the use of Cannabis associated with periodontitis? A systematic review and meta-analysis. J Periodontal Res. 2019. https://www.ncbi. nlm.nih.gov/pubmed/30677134

15. Lorosa, A.H., Pereira, C.M., Hussne, R.P., SilvaBoghossian, C.M. Evaluation of dental students' 
knowledge and patient care towards HIV/ AIDS individuals. Eur J Dent Educ. 2019. https:// www.ncbi.nlm.nih.gov/pubmed/30681237

16. Erchick, D.J., Rai, B., Agrawal, N.K., Khatry, S.K., Katz, J., LeClerq, S.C., et al. Oral hygiene, prevalence of gingivitis, and associated risk factors among pregnant women in Sarlahi District, Nepal. BMC Oral Health. 2019;19(1):2. https://www.ncbi.nlm.nih.gov/ pubmed/30611255

17. Shah, R., Kumbhalwar, A., Vyas, K., Raval, C., Kothari, R., Jinwala, M. Stress And Periodontal Disease: A Review. JAMDSR. 2016;4(3). http:// jamdsr.com/uploadfiles/16.Stress\&Periodontal DiseaseAReview.20160510081918.pdf

18. Bascones-Martínez, A., Escribano-Bermejo, M. Necrotizing periodontal disease: a manifestation of systemic disorders. Med Clin (Barc). 2005;15(125):706-13. https://www.ncbi.nIm.nih. gov/pubmed/16324485

19. Mannem, S., Chava, V.K. The effect of stress on periodontitis: A clinicobiochemical study. J Indian Soc Periodontol. 2012;16(3):3659. https://www.ncbi.nlm.nih.gov/pmc/articles/ PMC3498705/

20. Fenol, A., Jebi, S., Krishnan, S., Perayil, J., Vyloppillil, R., Bhaskar, A., et al. Association of stress, salivary cortisol level, and periodontitis among the inmates of a central prison in Kerala. Dent Res J (Isfahan). 2017;14(4):288-92. https:// www.ncbi.nlm.nih.gov/pubmed/28928784

21. Araujo, A.A., Morais, H.B., Medeiros, C., Brito, G.A.C., Guedes, P.M.M., Hiyari S, et al. Gliclazide reduced oxidative stress, inflammation, and bone loss in an experimental periodontal disease model. J Appl Oral Sci. 2019;27:e20180211. https://www.ncbi.nlm.nih. gov/pubmed/30810635

22. Sudhanshu, A., Sharma, U., Vadiraja, H.S., Rana, R.K., Singhal, R. Impact of Yoga on Periodontal Disease and Stress Management. Int J Yoga. 2017;10(3):121-7. https://www.ncbi.nlm. nih.gov/pubmed/29422742

23. Seraphim, A.P., Chiba, F.Y., Pereira, R.F., Mattera, M.S., Moimaz, S.A., Sumida, D.H. Relationship among Periodontal Disease, Insulin Resistance, Salivary Cortisol, and Stress Levels during Pregnancy. Braz Dent
J. 2016;27(2):123-7. https://www.ncbi.nlm.nih. gov/pubmed/27058372

24. Lu, H., Xu, M., Wang, F., Liu, S., Gu, J., Lin, S. Chronic stress enhances progression of periodontitis via alpha1-adrenergic signaling: a potential target for periodontal disease therapy. Exp Mol Med. 2014;46:e118. https://www.ncbi. nlm.nih.gov/pubmed/25323788

25. Goyal, S., Gupta, G., Thomas, B., Bhat, K.M., Bhat, G.S. Stress and periodontal disease: The link and logic!! Ind Psychiatry J. 2013;22(1):4-11. https://www.ncbi.nlm.nih.gov/ pubmed/24459366

26. Sondhi, P., Bhatia, V., Mehta, S. Response to article: Stress and periodontal disease: The link and logic!! Ind Psychiatry J. 2013;22(2):165. https://www.ncbi.nlm.nih.gov/ pubmed/25013323

27. Ozberk, S.S., Gundogar, H., Senyurt, S.Z., Erciyas, K. Adjunct Use of Low-Level Laser Therapy on the Treatment of Necrotizing Ulcerative Gingivitis: A Case Report. J Lasers Med Sci. 2018;9(1):73-5. https://www.ncbi.nlm.nih.gov/ pubmed/29399316

28. Berres, F., Marinello, C.P. [Necrotizing ulcerative periodontitis. Diagnosis, treatment and follow-up--a case report]. Schweiz Monatsschr Zahnmed. 2004;114(5):479-95. https://www. ncbi.nlm.nih.gov/pubmed/15250177

29. Kato, H., Imamura, A. Unexpected Acute Necrotizing Ulcerative Gingivitis in a Wellcontrolled HIV-infected Case. Intern Med. 2017;56(16):2223-7. https://www.ncbi.nIm.nih. gov/pubmed/28781315

30. Hu, J., Kent, P., Lennon, J.M., Logan, L.K. Acute necrotising ulcerative gingivitis in an immunocompromised young adult. BMJ Case Rep. 2015;2015. https://www.ncbi.nlm.nih.gov/ pubmed/26376700

31. Anjum, I., Jaffery, S.S., Fayyaz, M., Wajid, A., Ans, A.H. Sugar Beverages and Dietary Sodas Impact on Brain Health: A Mini Literature Review. Cureus. 2018;10(6):e2756. https://www. ncbi.nlm.nih.gov/pubmed/30094113

32. Stults-Kolehmainen, M.A., Sinha, R. The effects of stress on physical activity and exercise. Sports Med. 2014;44(1):81-121. https://www. ncbi.nlm.nih.gov/pubmed/24030837 
33. Murta, S.G., Tróccoli, B.T. Intervenções psicoeducativas para manejo de estresse ocupacional: um estudo comparativo. Rev Bras de Ter Comp Cogn. 2009;11(1):25-42. http://www. usp.br/rbtcc/index.php/RBTCC/article/view/382

34. Falkler, W.A.E.C.O., Idigbe, E.O. Microbiological understandings and mysteries of noma

(cancrum oris). Oral Dis. 2008;5(2)150-5. https:// www.ncbi.nlm.nih.gov/pubmed/10522213

35. SHANNON, I.L.K., O'LEARY, T. J. Stress as a Predisposing Factor in Necrotizing Ulcerative Gingivitis. J Periodontol. 1969;40(4):240-2. https://www.ncbi.nlm.nih.gov/pubmed/5253993

36. Hildebrand, H.C., Epstein, J., Larjava, H. The influence of psychological stress on periodontal disease. J West Soc Periodontol Periodontal Abstr. 2000;48(3):69-77. https://www.ncbi.nlm. nih.gov/pubmed/11381953

37. Herrera, D., Retamal-Valdes, B., Alonso, B., Feres, M. Acute periodontal lesions (periodontal abscesses and necrotizing periodontal diseases) and endo-periodontal lesions. J Clin Periodontol. 2018;45 Suppl 20:S78-S94. https:// www.ncbi.nlm.nih.gov/pubmed/29926493

38. Corbet, E.F. Diagnosis of acute periodontal lesions. Periodontol 2000. 2004;34:20416.

https://www.ncbi.nlm.nih.gov/ pubmed/14717863

39. Yang, C.L., Chen, C.H. Effectiveness of aerobic gymnastic exercise on stress, fatigue, and sleep quality during postpartum: A pilot randomized controlled trial. Int J Nurs Stud. 2018;77:1-7. https://www.ncbi.nlm.nih.gov/ pubmed/28950158

Submetido em: 3-10-2018

Aceito em: 21-6-2019 